\title{
Corneal elastosis within lattice dystrophy lesions
}

\author{
JACOB PE'ER,' BEN S FINE, , ANITA DIXON, ${ }^{3}$ AND DAVID S ROTHBERG \\ From the 'Department of Ophthalmology, Hadassah University Hospital, Jerusalem, Israel, ${ }^{2}$ Department of \\ Ophthalmic Pathology, Armed Forces Institute of Pathology, Washington, DC, and the Departments of \\ ${ }^{3}$ Pathology and ${ }^{4}$ Ophthalmology, Veterans Administration Hospital, Kansas City, Missouri, USA
}

SUMMARY Corneal buttons of two patients with lattice corneal dystrophy were studied by light and electron microscopy. They showed elastotic degeneration within the amyloid deposits. The amyloid deposits displayed characteristic staining; the elastotic material (elastin) within the deposits stained positive with Verhoeff-van Gieson and Movat pentachrome stains and showed autofluorescence. The characteristic ultrastructural findings of amyloid and elastotic material were also demonstrated. The possibility of the associations of these two materials in the cornea is discussed.

Corneal elastosis, also known by a wide variety of synonyms ${ }^{1-6}$ may be seen as a single corneal or ocular finding, or in association with a variety of chronic ocular and corneal disorders such as scarring secondary to chemical or mechanical trauma, glaucoma, actinic lesions, old inflammation, corneal oedema, conjunctival carcinoma in situ or invasive squamous cell carcinoma, and ochronosis. ${ }^{78}$ Corneal elastosis has been described in association with lattice corneal dystrophy in a few previous reports. ${ }^{28-11}$ In one of these cases the elastosis was in close relation to the amyloid, ${ }^{10}$ and in two cases the elastosis was found within the amyloid. ${ }^{89}$ In the last two there was no detailed description.

The subjects of this report are two patients with lattice corneal dystrophy in whose corneas we found elastosis within the amyloid deposits. This finding was demonstrated by light and electron microscopy and by an autofluorescent technique.

\section{Case reports}

CASE 1

A 63-year-old white male had a 15-year history of decreasing visual acuity in both eyes because of corneal opacities that became worse in the four months before his operation. Cataracts had progressed rapidly in the previous six months.

There was no known family history of any eye

Correspondence to Jacob Pe'er, MD, Department of Ophthalmology, Hadassah University Hospital, PO Box 12000, 91120 Jerusalem, Israel. disease other than myopia. The patient was admitted to hospital in January 1983 for examination of a lung nodule, but, because he refused bronchoscopy, a definite diagnosis was not made. There was history of alcohol abuse in the past, but that had been stopped for several months.

In February 1983 the patient was admitted to hospital for penetrating keratoplasty of the right eye, cataract extraction, and posterior chamber lens implantation. Examination of the eyes revealed visual acuity of hand motion in each eye. The intraocular pressures were within normal limits. The

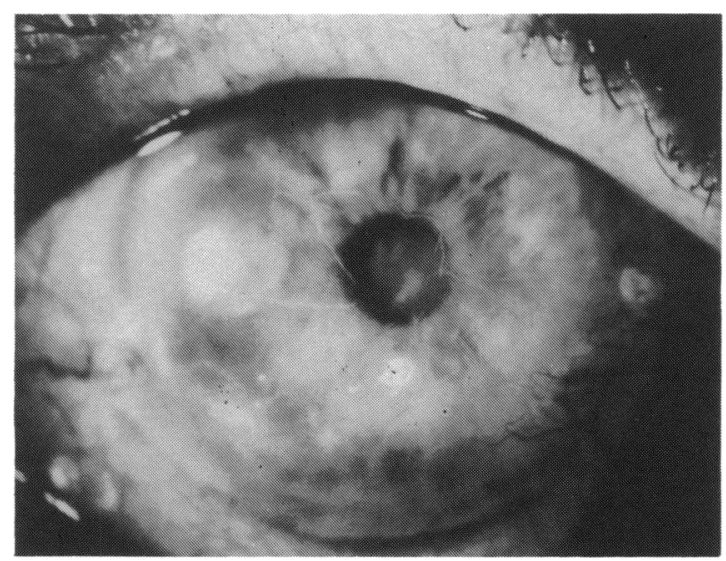

Fig. 1 Case 1. Right eye shows semitranslucent dichotomous refractile lines and some white dots in the cornea. 


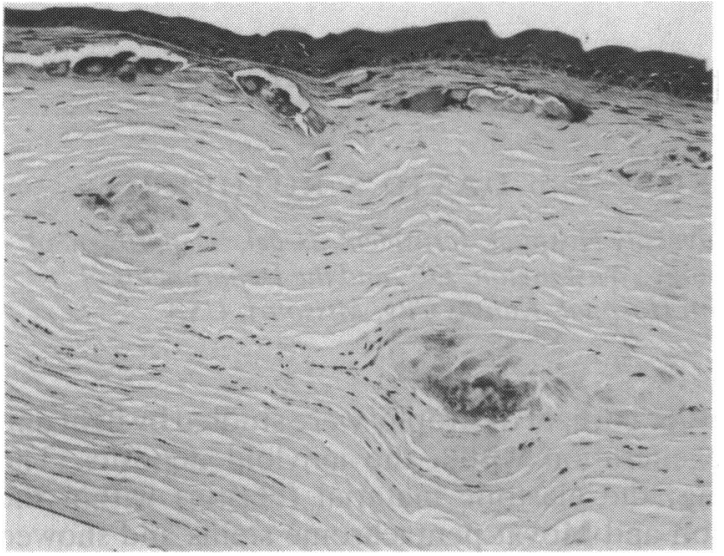

Fig. 2 Case 1. Histological section of the cornea shows uneven epithelium, fibrotic pannus and replacement of Bowman's membrane by fibrous tissue, and basophilic hyaline nodules. In the stroma there are fusiform deposits of amyloid, with hyaline deposits within them. Haematoxylineosin.

pupils reacted normally to light. Slit-lamp examination revealed many semitranslucent, dichotomous, refractile lines and some white dots in both corneas consistent with lattice corneal dystrophy (Fig. 1). There were small pterygia in both eyes. The anterior chambers were deep and clear. Funduscopic examination could not be done owing to the dense cataracts. Ultrasonographic examination was normal. After the planned surgical procedure was performed, the patient did well, his vision being $6 / 120$ without correction in the right eye, corrected to $6 / 24$ with pinhole. The graft remained clear.

Pathological findings. Macroscopic examination

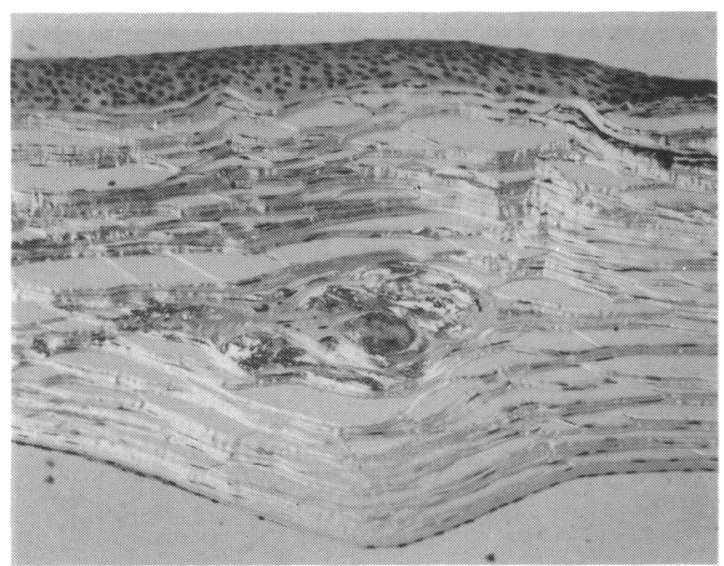

Fig. 3 Case 1. The amyloid fusiform material shows birefringence with polarised light, while the hyaline deposits within it do not show this. Congo-red, polarised light.

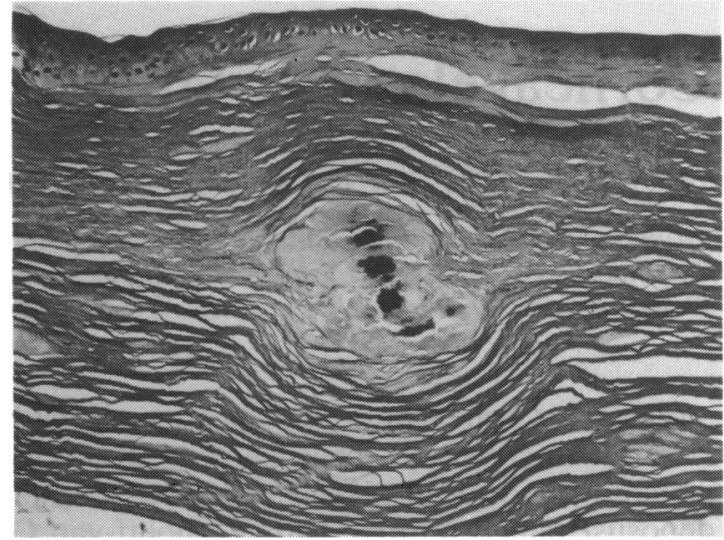

Fig. 4 Case 1. Black elastotic material within the amyloid deposits. Verhoeff-van Gieson.

revealed two pieces of tissue. One was labelled pterygium of the right eye and consisted of a single fragment of grey-brown soft tissue, measuring $3 \times 2 \times 2 \mathrm{~mm}$. The entire specimen was submitted for light microscopic examination. The other piece of tissue was a translucent corneal button, $7 \mathrm{~mm}$ in diameter, with many semitranslucent lines in its stroma. A section of the cornea was submitted for electron microscopy, while the remainder was submitted for light microscopy.

Microscopic examination of the soft tissue revealed conjunctiva with focal squamous metaplasia, hyalinisation and elastotic degeneration of collagen, and some chronic inflammation, an appearance consistent with pterygium.

Microscopic examination of the cornea revealed uneven epithelium, fibrotic pannus in one area, and replacement of Bowman's membrane by fibrous tissue and basophilic hyaline nodules in some areas (Fig. 2). In the anterior two-thirds of the stroma there

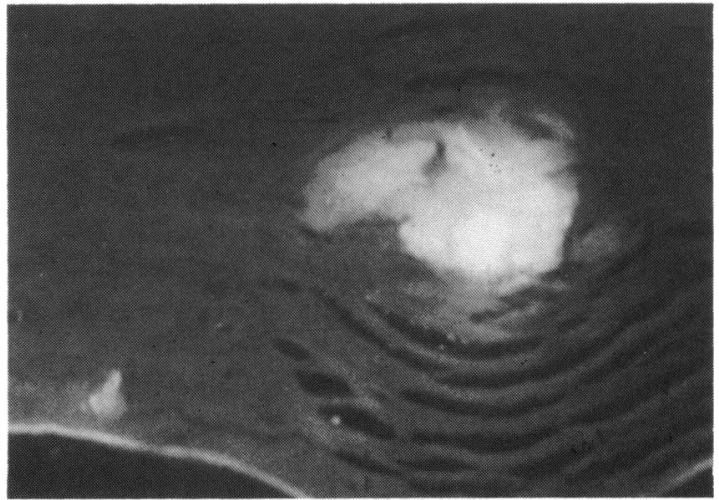

Fig. 5 Case 1. The elastotic material shows strong autofluorescence. Autofluorescence. 


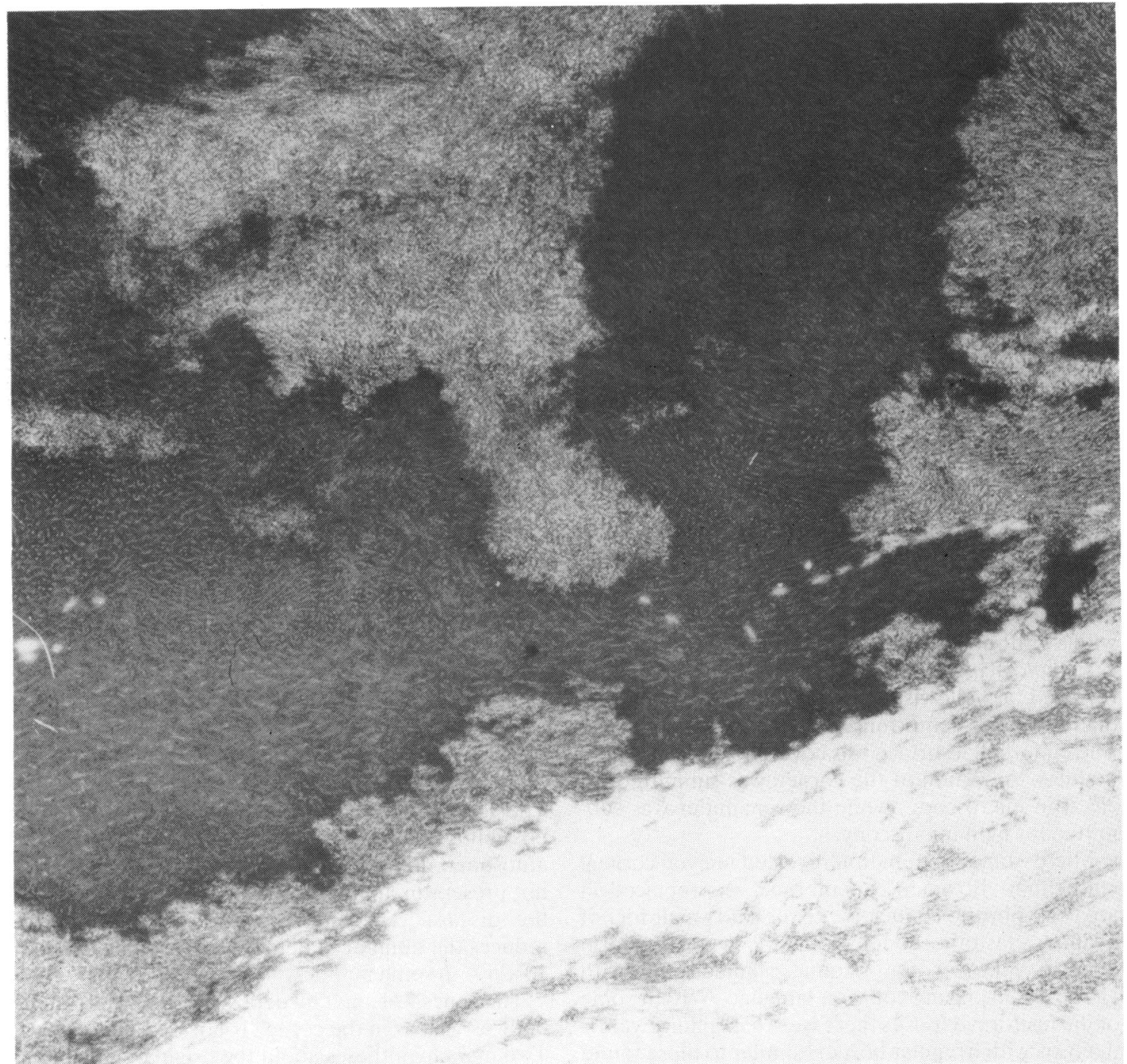

Fig. 6 Case 1. Ultrastructurally the amyloid material (lighter) shows delicate fibrils, mostly non-branching, without periodicity. The elastotic material is electron-dense with irregular edges, showing delicate fibrillar pattern. Electron micrograph.

were fusiform deposits, stained pink with haematoxylin and eosin ( $\mathrm{H}$ and $\mathrm{E})$, displacing collagen lamellae. Within many of these fusiform deposits there were blue-grey hyaline deposits with irregular borders similar to those found in Bowman's membrane and the anterior stromal areas (Fig. 2). The intervening stroma appeared normal except for some areas of stromal oedema. Descemet's membrane was unremarkable, and the endothelial cells were attenuated. The fusiform deposits were stained deep purple with periodic acid-Schiff (PAS), red-purple with Masson's trichrome stain, pink-purple with Movat pentachrome stain, and yellow-grey with Verhoeffvan Gieson (VVG) elastin stain. Congo red stained the deposits orange; it showed dichroism of green and red and birefringence with polarised light, more than the surrounding stroma and consistent with amyloid material. The hyaline deposits within the amyloid did not show these appearances (Fig. 3). The hyaline deposits were stained pink-grey with PAS, blue-purple with Masson trichrome, blue-grey with Congo red, and black with VVG and Movat pentachrome stains, consistent with elastotic material (Fig. 4). Von Kossa stain for calcium was negative. These 
deposits were autofluorescent with ultraviolet light (Fig. 5).

Ultrastructurally the amyloid lesions showed delicate, mostly non-branching fibrils without periodicity, many of them highly aligned. Within this material there were electron-dense deposits with irregular edges consistent with elastin-like material and showing a delicate fibrillar pattern that resembled the amyloid pattern (Fig. 6).

CASE 2

A 49-year-old Asian Indian male known to have tuberculoid leprosy had suffered for the last four years a gradual, slowly progressive diminution of vision in both eyes. He gave a history of injury with a grass plant to the right eye four years previously. His general condition was good. There was no family history of eye disease. On examination his visual acuity was $4 / 60$ in the right eye and $6 / 60$ in the left eye, with intraocular pressure within normal limits. Slitlamp examination showed typical lattice corneal dystrophy lines in both eyes. In February 1983 he underwent penetrating keratoplasty of his right eye, with a graft of $7.5 \mathrm{~mm}$ in diameter.

Pathological findings. Macroscopic examination revealed a yellowish corneal button $7.5 \mathrm{~mm}$ in diameter with semi-translucent lines in its stroma. The endothelial surface was covered with pigmented nodules. A section of the cornea was submitted for electron microscopy, while the remainder was submitted for light microscopy.

Microscopic examination revealed uneven corneal epithelium. Bowman's membrane was replaced in areas by fibrotic connective tissue and small foci of hyaline elastotic nodules. There were fusiform deposits all over the stroma, stained pink with $\mathrm{H}$ and $\mathrm{E}$, displacing collagen lamellae. Within some of the fusiform deposits there were basophilic hyaline deposits with irregular borders similar to those found in Bowman's membrane and anterior stromal areas. The intervening stroma appeared normal. Descemet's membrane was unremarkable. The endothelial cells were mildly attenuated. The posterior surface of the cornea was severely overgrown with a pigmented fungus which we interpreted as a contaminant. Special stains gave the same results as in case 1. The fusiform deposits were stained orange with Congo red and showed dichroism and birefringence with polarised light more than the surrounding stroma, consistent with amyloid material. The hyaline deposits within them did not show these appearances. They stained black with VVG and Movat pentachrome stains (Fig. 7) and were autofluorescent with ultraviolet light, consistent with elastotic material.

Ultrastructurally the amyloid lesions showed

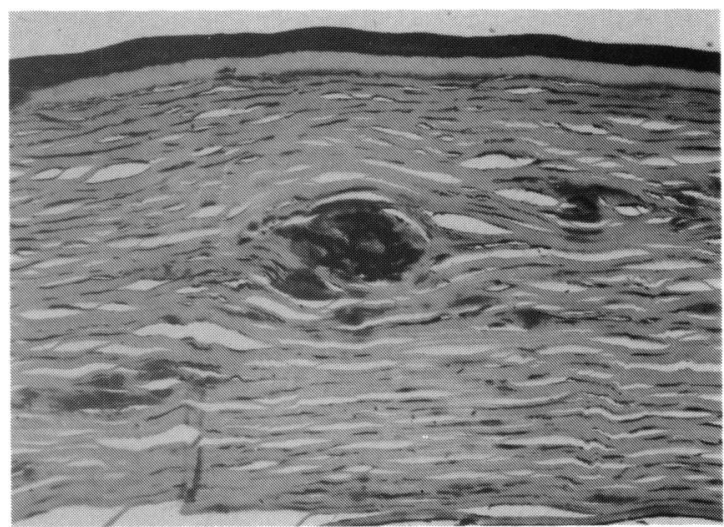

Fig. 7 Case 2. Fusiform amyloid deposit in the stroma with elastotic material stained black within it. Movat pentachrome.

delicate, mostly non-branching fibrils without periodicity. Within this material there were electrondense deposits with irregular edges consistent with elastin-like material, the same findings as in case 1 (Fig. 8).

\section{Discussion}

The clinical and histological findings in our cases are compatible with lattice dystrophy of the cornea. The lack of family history does not rule out this possibility. Although lattice dystrophy has an autosomal dominant pattern of inheritance, it does not present in all affected members of families with the disease. The subtle expression of the gene reduces the number of detected individuals to about $10-25 \%$ in some families. ${ }^{112}$

The genesis of amyloid deposition elsewhere in the body as well as in the cornea is not well understood. Two main hypotheses about the origin of amyloid in lattice dystrophy are: (1) collagen degeneration caused by lysosomal enzymes released from abnormal keratocytes, ${ }^{12-14}$ and (2) synthesis of amyloid by keratocytes. ${ }^{12-15}$ Other theories consider the lattice as focal amyloidosis restricted to the cornea, ${ }^{16}$ as primary degeneration of corneal nerves, ${ }^{17}$ or as related to amyloid protein AA, a precursor protein possibly attached to a transport protein. ${ }^{18}$

The current concept of the pathogenesis of corneal elastosis is that it is a result of collagen fibre degeneration. ${ }^{7419211}$ The resistance of the elastotic material to digestion with elastase ${ }^{11}$ and its autofluorescent characteristic show its differences from elastin and its similarity to elastotic degeneration elsewhere, as in the conjunctiva. Another study, based on some tinctorial dissimilarities between 


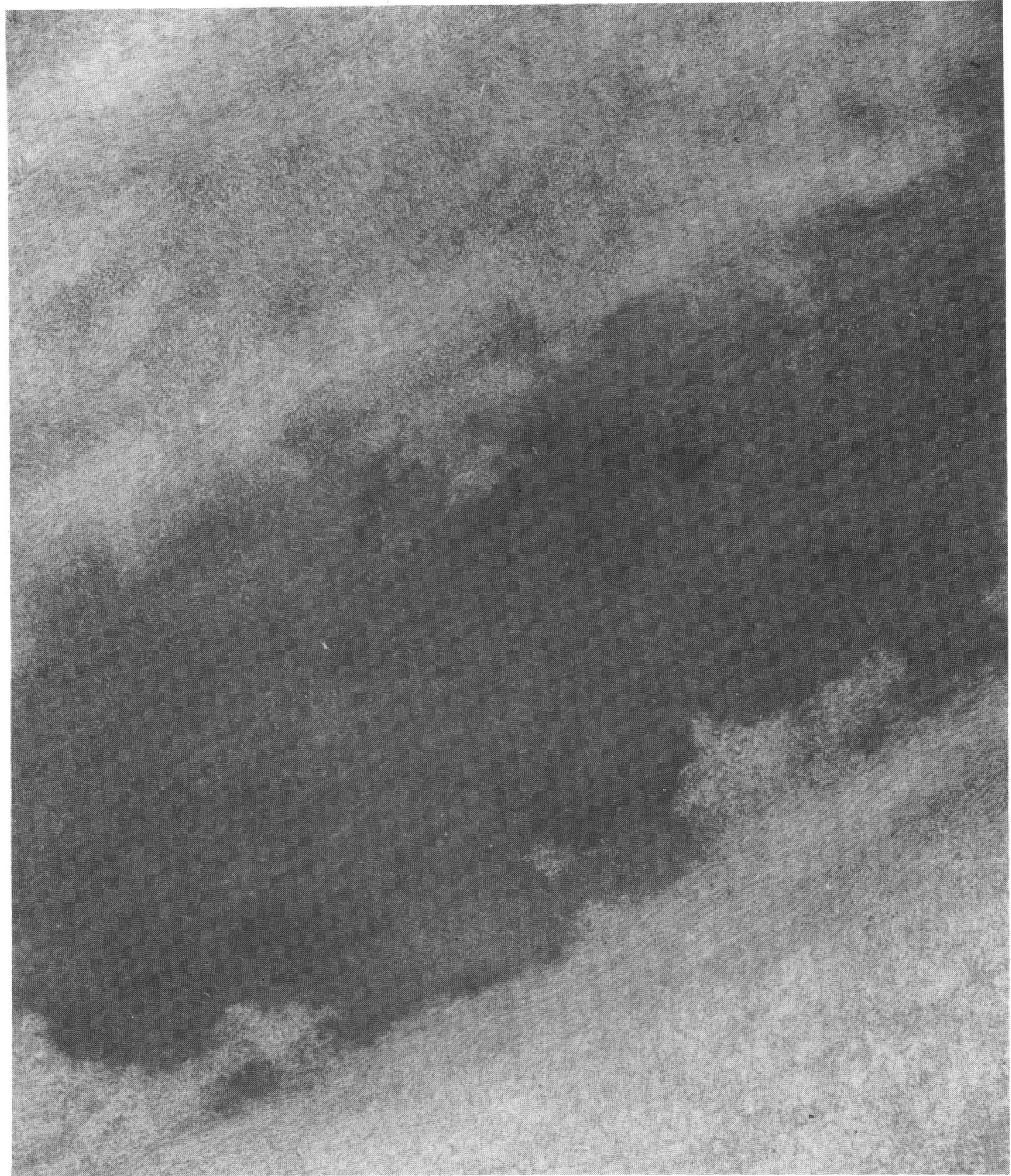

Fig. 8 Case 2. Ultrastructurally the amyloid material (lighter) is composed of delicate, mostly non-branching fibrils without periodicity. The elastotic material is electron-dense, with irregular borders, showing delicate fibrillar pattern. Electron micrograph.

pseudoelastic fibres in pingueculae and spheroidal elastotic deposits in the cornea, suggests that the spheroidal elastotic deposits might be a composite of degenerate collagen and a second non-collagenous protein. ${ }^{21}$ The latter may be of altered plasma constituents from leaking limbal vessels. ${ }^{22}$

The accepted theories of collagen degeneration in both lattice dystrophy and corneal elastosis suggest 
the possibility of a closely related metabolic reason for both, although one is genetically inherited and the other is not. Corneal elastosis may be associated with a variety of chronic ocular and corneal disorders. ${ }^{7 \times}$ Our patients presented clinical findings or a history of other corneal diseases like those of other cases with corneal elastosis. Case 1 had pterygium in both eyes. The main characteristic of pterygium is elastotic degeneration of the conjunctival collagen fibres, and cases of pterygium also sometimes show elastotic degeneration of the cornea. Case 2 presented a history of old trauma to the cornea, a common feature of other cases of elastotic degeneration. We can conclude that in these two cases of lattice dystrophy there were other causes of the elastotic degeneration. The combined finding of lattice dystrophy and elastosis was described in previous reports, ${ }^{2 \times-10}$ so our earlier explanations can account for this combination but not the existence of the elastotic material within the amyloid, as in our cases.

The opinions or assertions contained herein are the private views of the authors and are not to be construed as official or as reflecting the views of the Department of the Army or the Department of Defence.

\section{References}

1 Freedman A. Labrador keratopathy. Arch Ophthalmol 1965; 74: 198-202.

2 Garner A. Keratinoid corneal degeneration. Br J Ophthalmol 1970; 54: 769-80.

3 Klintworth G. Chronic actinic keratopathy. Am J Pathol 1972 ; 67: $327-42$.

4 Fraunfelder FT, Hanna C, Parker JM. Spheroid degeneration of cornea and conjunctiva. 1. Clinical course and characteristics. Am J Ophthalmol 1972; 74: 821-8.

5 Fraunfelder FT, Hanna C, Parker JM. Spheroid degeneration of cornea and conjunctiva. 2. Pathology. Am J Ophthalmol 1972; 74: 829-39.
6 Freedman A. Climatic droplet keratopathy. 1. Clinical aspects Arch Ophthalmol 1973; 89: 193-7.

7 Brownstein S, Rodrigues MM, Fine BS, Albert AN. The elastotic nature of hyaline corneal deposits. Am J Ophthalmol 1973; 75: 799-809.

8 Fraunfelder FT, Hanna C. Spheroidal degeneration of cornea and conjunctiva. 3 . Incidences, classification and etiology. A $\mathrm{m} \mathrm{J}$ Ophthalmol 1973; 76: 41-50.

9 Rodrigues MM, Laibson PR, Weinreb S. Corneal elastosis. Arch Ophthalmol 1975; 93: 111-4.

10 Dubord PJ, Rodrigues MM, Krachmer JH. Corneal elastosis in lattice corncal dystrophy. Ophthalmology 1981 ; 88: 1239-43.

11 Ramsey RM. Familial corneal dystrophy lattice type. Trans Am Ophthalmol Soc 1957; 60: 701-39.

12 Dark AJ, Thompson DS. Lattice dystrophy of the cornea: a clinical and microscopic study. $\mathrm{Br} J$ Ophthalmol 1960; 44: 257-79.

13 Klintworth GK. Current concepts on the ultrastructural pathogenesis of macular and lattice corneal dystrophies. Birth Defects (Orig Art Ser) 1971; 7: 27-31

14 Waring GO III, Rodrigues MM, Laibson PR. Corneal dystrophies: 1. Dystrophies of the epithelium, Bowman's layer and stroma. Surv Ophthalmol 1978; 23: 71-122.

15 Yanoff M, Fine BS, Colosi NJ, Katowitz JA. Lattice corneal dystrophy. Report of an unusual case. Arch Ophthalmol 1977; 95: $651-5$.

16 Klintworth GK. Lattice corneal dystrophy, an inherited variety of amyloidosis restricted to the cornea. Am J Pathol 1967; 50: 371-99.

17 Walter JR, Henderson JW. Lattice dystrophy of the cornea: a primary hyaline degeneration of corneal nerves and superficial stroma cells. Trans Am Ophthalmol Soc 1962; 60: 176-204.

18 Mondino BJ, Sundar Raj CV, Skinner M, Cohen AS. Protein AA and lattice corneal dystrophy. Am J Ophthalmol 1980; 89: 377-80.

19 Garner A, Morgan G, Tripathi RC. Climatic droplet keratopathy. II. Pathologic findings. Arch Ophthalmol 1973; 89: 198-204.

20 Cursino JW, Fine BS. A histologic study of calcific and noncalcific band keratopathies. Am J Ophthalmol 1976; 82: 395-404.

21 Garner A, Fraunfelder FT, Barras TC, Hinzpeter EN. Spheroidal degeneration of cornea and conjunctiva. $\mathrm{Br} J$ Ophthalmol 1976; 60: 473-8.

22 Johnson GJ, Overall M. Histology of spheroidal degeneration of the cornea in Labrador. Br J Ophthalmol 1978; 62: 53-61.

Accepted for publication 30 December 1986. 